\title{
Improving the Performance of All-Optical Logic Gates Based on Metal-Insulator-Metal Heterogeneous Plasmonic Waveguide by Kerr Nonlinearity Effect
}

\section{Mojtaba Kamali}

Islamic Azad University Najafabad Branch

Mehdi Riahinasab ( $\nabla$ m.riahinasab@pel.iaun.ac.ir)

Islamic Azad University Najafabad Branch https://orcid.org/0000-0003-1059-0510

\section{Zahra Alaie}

Islamic Azad University Najafabad Branch

\section{Research Article}

Keywords: All-optical logic gate, Plasmonic waveguide, Kerr effect.

Posted Date: January 20th, 2022

DOI: https://doi.org/10.21203/rs.3.rs-1265299/v1

License: (c) (1) This work is licensed under a Creative Commons Attribution 4.0 International License.

Read Full License 


\title{
Improving the Performance of All-Optical Logic Gates Based on Metal- Insulator-Metal Heterogeneous Plasmonic Waveguide by Kerr Nonlinearity Effect
}

\author{
Mojtaba Kamali ${ }^{1}$, Mehdi Riahinasab ${ }^{1,2, *}$, Zahra Alaie ${ }^{1,3}$ \\ 1 Department of Electrical Engineering, Najafabad Branch, Islamic Azad University, Najafabad, Iran \\ 2 Smart Microgrid Research Center, Najafabad Branch, Islamic Azad University, Najafabad, Iran \\ ORCID ID: 0000-0003-1059-0510 \\ 3 Digital Processing and Machine Vision Research Center, Najafabad Branch, Islamic Azad University, \\ Najafabad, Iran \\ ORCID ID: 0000-0002-7799-5152 \\ *Corresponding Author: Mehdi Riahinasab (m.riahinasab@pel.iaun.ac.ir)
}

\begin{abstract}
In this paper, the nonlinear effect of Kerr on increasing the efficiency of multi-channel alloptical metal-insulator-metal (MIM) logic gates with heterogeneous metals is investigated. In this study, linear interference between surface plasmonic polariton modes has been employed. The performance of OR, AND, XOR and NOR gates are numerically analyzed by the finite element method (FEM). A structure with fixed physical dimensions can manage several basic logic functions. By applying optical signals to various input ports in the proposed structure, we can implement the logic functions of OR, AND and XOR. The NOR logic gate can be established by the addition of a control optical signal. On the other hand, to improve the level difference between the logic states of " 0 " and " 1 " in the waveguides, heterogeneous metals as well as Kerr material with non-linear effect have been employed. It has been shown that the nonlinear material of Kerr increases the difference of field intensity between logic levels of " 0 " and "1" are $77 \mathrm{~V} / \mathrm{m}$ for OR gate, $133 \mathrm{~V} / \mathrm{m}$ for AND gate, $89 \mathrm{~V} / \mathrm{m}$ for XOR gate and $138 \mathrm{~V} / \mathrm{m}$ for NOR gate. The proposed all-optical logic gate has a simple and compact arrangement and it can be applied to many nanophotonic components used in optical communication networks.
\end{abstract}

Keywords All-optical logic gate, Plasmonic waveguide, Kerr effect.

\section{Introduction}

All-optical logic gates are one of the most significant components in optical networks (Caulfield and Dolev 2010). One of the challenges in designing of optical logic gates is the issue of light interference, which is due to the wave property of light. The logic gates based on linear optical interference usually have a small difference in intensity between the two logic levels of " 0 " and "1" (Li et al. 2005 and Zhang et al. 2007). The logic operations depend on the optical phase ratio of the two input signals and accurate control of optical phase deference is not easy and the creation of constructive or destructive interferential modes of signals is almost difficult (Sankar and Philip 2018). By downsizing the device such as photonic crystal structures (Fu et al. 2013), this problem 
can be solved. However, compact interfering logic gates based on these structures cannot have physical dimensions in the subwavelength scale due to diffraction limitation.

Reciprocally, superficial plasmon structures can limit or transmit light in the subwavelength range (Onuki et al. 2003 and Moradipour et al. 2021). So far, many examples of such plasmonic interfering structures based on metal nanowire networks and metal slot waveguides have been investigated (Lu and Pan 2013 and Papaioannou 2016). However, in order to implement all basic logic functions, different structures are needed which making the design and application of these components complex.

To solve this limitation, a suitable all-optical logic device based on plasmonic waveguides has been presented (Bian and Gong 2014). By using numerical simulation of the proposed structure at wavelength $1550 \mathrm{~nm}$, the basic logic gates OR, AND, NOT, XOR, XNOR, NAND and NOR have been implemented, without any modification or reconfiguration the device. In this design, a general logic device is constructed using metal-insulator-metal waveguide (MIM).

In this design, to increase the level difference between the logic states of " 0 " and " 1 ", heterogeneous metals of gold, aluminum, and silver as well as Kerr material with nonlinear effect have been used for the basic logic gates of OR, AND, XOR and NOR (Ghorbani 2020 and Lu 2011). The simulated gates have a simple structure, nanoscale light confinement, minimal interference, acceptable propagation distance and very high transmission (Bian 2013, Dionne 2006 and $\mathrm{Xu}$ 2014). The logic gates based on metal-insulator-metal (MIM) can create logic functions by displacing optical signals through input and control channels. Also, we show that a high difference level is obtained between the logic levels of " 0 " and "1".

\section{Design of all-optical logic gate based on metal-insulator-metal structure}

The two-dimensional structure of the first proposed design is shown in Fig. 1a. This structure consists of four air channels located in the substrate of silver, gold and aluminum as input ports and one output signal channel. The input signals are directed through ports $I_{1}, I_{2}$ and $I_{3}$. Port $C$ is also required as a control port for the creation of certain logic functions. The same thickness (d) is considered for all channels. The distance between all channels is constant and equal to L. All corners are considered with a sharp 90-degree angle. Permittivity of air, silver, gold, and aluminum are considered $\varepsilon_{A i r}=1 \quad, \quad \varepsilon_{A g}=-129+3.3 i \quad, \quad \varepsilon_{A u}=-113.53+9.268 i \quad$, and $\varepsilon_{A l}=-197.92+38.85 i$, respectively.

The results show that the real part of the modal effective index and the propagation losses of plasmonic modes decrease with an increase in channel thickness (Bian and Gong 2014). But the size of the corresponding mode increases. In order to establish single-mode conditions as well as acceptable propagation losses, we considered the thickness of the channels between $d=50 \mathrm{~nm}$ to $\mathrm{d}=100 \mathrm{~nm}$. The choice of this range is due to the fact that the real part of the effective index remains constant in considered thickness of the channels. Also, in order to reduce the interference between the input signals of the channels, the distance between all the channels must be greater than the critical value required to ensure the correct logic operations (Bian and Gong 2014). 
The two-dimensional geometry of the second proposed structure is shown in Fig. 1b. This structure is the same as the first one. Only at the outlet of channels $I_{1}$ and $I_{2}$ and their connection to channels $\mathrm{I}_{3}$ and $\mathrm{C}$, a Kerr nonlinear material with nonlinear coefficient of $1 \times 10^{16}$ and linear dielectric constant of 2.31 is used instead of air inside the waveguide (highlighted in red in the Fig. 1b. The optical specifications of logic gates based on metal-insulator-metal have been studied at $1550 \mathrm{~nm}$ by the FEM.

\section{Results and discussion}

The operation of optical logic gates is considered to be based on the wave property of light. If the phase difference between two light beams is $2 \mathrm{k} \pi$, a constructive interference will take place between the two beams, and creates a relatively high intensity output signal. However, if the phase difference between two light beams is $(2 \mathrm{k}+1) \pi$, destructive interference takes place and as a result, the output signal is obtained with an intensity of almost zero (Sankar and Philip 2018). With a correct definition of threshold intensity, the high and low logic levels can be accomplished based on the both constructive and destructive interference (Bian and Gong 2014). According to the proposed structure, the difference in the optical paths for different channels causes the constructive or destructive interference for the input optical signals. There is no difference in the optical path between the signals in channels $I_{1}$ and $I_{2}$, which leads to a zero-phase difference. While there is an optical path difference for channels $\mathrm{I}_{1}$ (or $\mathrm{I}_{2}$ ) and $\mathrm{I}_{3}$ (or $\mathrm{C}$ ) which is equal to half of the effective wavelength of the guided plasmonic modes (Bian and Gong 2014), a phase difference is brought about in $\pi$. Therefore, we have constructive interference for the signals of channels $\mathrm{I}_{1}$ and $\mathrm{I}_{2}$; while the signal in channels $\mathrm{I}_{3}$ and $\mathrm{C}$ creates a destructive interference with channels $I_{1}$ and $I_{2}$ in the output. With regard to the above-mentioned considerations and simulations, in order to reduce the interference between the input signals as well as the required constructive and destructive interferences, the optimal distance between the channels has been considered $\mathrm{L}=550 \mathrm{~nm}$.

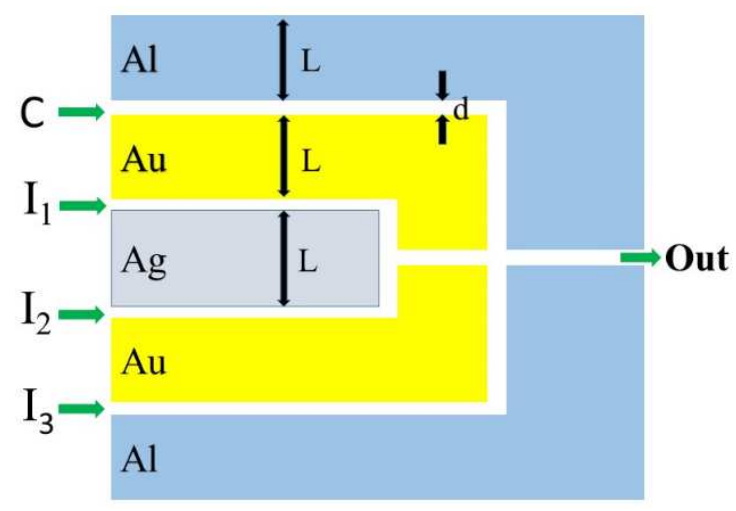

(a)

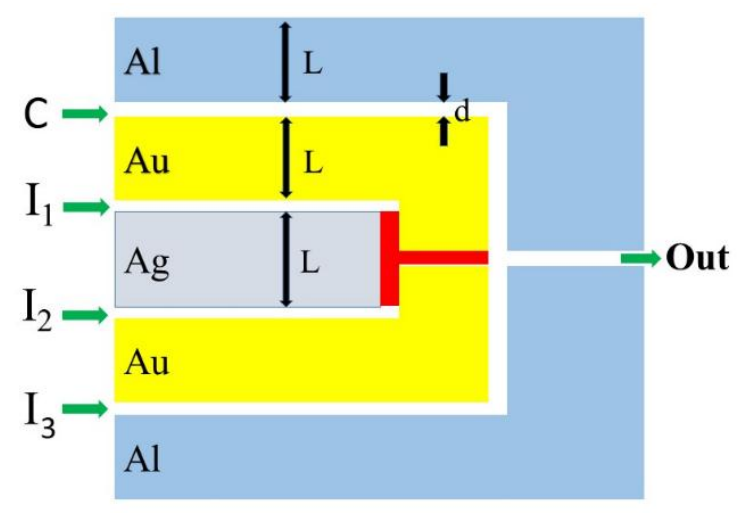

(b)

Fig. 1 Geometry of proposed structures for all-optical logic gate using (a) three different metals, (b) three different metals and Kerr nonlinear material. 
To perform different logic operations, there is a need for the combination of two or more channels in the logic gate. Table 1 shows the combinations of entered signal to different channels for the OR, AND, XOR, and NOR logic gates.

Implementation of OR, AND, and XOR logic gates can be activated by combining the two input channels. By applying constructive interference between the $I_{1}$ and $I_{2}$ signals and correctly defining the threshold intensity, OR and AND logic gates can be accomplished. Also, by applying destructive interference between the $\mathrm{I}_{2}$ and $\mathrm{I}_{3}$ signals, the XOR gate can be performed. On the other hand, in order to accomplish the NOR logic function, the signal in the control channel must also be activated. For NOR gate, both the channel signals of $I_{1}$ and $I_{2}$ must be combined with the $\mathrm{C}$ control signal. In the NOR gate, the intensity of the control signal must be twice as great as the input signals. By correct determination of the threshold intensity, the NOR gate can be performed.

Table 1 The combination of entered signals to different channels for the performance related to logic operation: OR (constructive interference between $\mathrm{I}_{1}$ and $\mathrm{I}_{2}$ ), AND (constructive interference between $\mathrm{I}_{1}$ and $\mathrm{I}_{2}$ - The appropriate threshold field intensity should be defined for the gate to be on or off), XOR (destructive interference between $\mathrm{I}_{2}$ and $\mathrm{I}_{3}$ ) and NOR (destructive interference between $\mathrm{I}_{1}$ (and $\mathrm{I}_{2}$ ) with C)

\begin{tabular}{|c|c|c|c|c|}
\hline Gate & $\begin{array}{c}\text { Input } \\
\text { Channel } \mathrm{I}_{1}\end{array}$ & $\begin{array}{c}\text { Input } \\
\text { Channel } \mathrm{I}_{2}\end{array}$ & $\begin{array}{c}\text { Input } \\
\text { Channel } \mathrm{I}_{3}\end{array}$ & $\begin{array}{c}\text { Input } \\
\text { Channel C }\end{array}$ \\
\hline OR & Enabled & Enabled & & \\
\hline AND & Enabled & Enabled & & \\
\hline XOR & & Enabled & Enabled & \\
\hline NOR & Enabled & Enabled & & Enabled \\
\hline
\end{tabular}

\subsection{The OR gate performance}

The performance of the OR gate operation is shown in Table 2. By applying an optical signal to the input ports of channels $I_{1}$ and $I_{2}$ and without any requirement to activate the control signal, the output logic levels can be changed from "0" to "1". Fig. 2 shows the distribution of the magnetic field for various logic states.

Table 2 The procedure of using gate channels to implement the OR logic gate

\begin{tabular}{|c|c|c|c|c|c|}
\hline Gate & $\begin{array}{c}\text { Input } \\
\text { Channel I }\end{array}$ & $\begin{array}{c}\text { Input } \\
\text { Channel } I_{2}\end{array}$ & $\begin{array}{c}\text { Input } \\
\text { Channel I }\end{array}$ & $\begin{array}{c}\text { Input } \\
\text { Channel C }\end{array}$ & $\begin{array}{c}\text { Output } \\
\text { Channel }\end{array}$ \\
\hline \multirow{3}{*}{ OR } & "0" & "0" & & Disable & "0" \\
\cline { 2 - 6 } & "0" & "1" & & Disable & "1" \\
\cline { 2 - 6 } & "1" & "0" & & Disable & "1" \\
\cline { 2 - 6 } & & "1" $1 "$ & & Disable & "1" \\
\hline
\end{tabular}


According to Fig. 2(a,b), if a signal enters the $I_{1}$ or $I_{2}$ channels, this optical signal can be transmitted through the associated channel and appears at the output port corresponding to the logic operation " 1 OR $0=1$ " or " 0 OR $1=1$ ". On the other hand, if the signal is injected simultaneously into both the input ports of channels $I_{1}$ and $I_{2}$ (Fig. 2c), the optical signal can be transmitted through the both channels and reach the output channel in a combined form. This is because of channels $I_{1}$ and $I_{2}$ have a similar light path, therefore making the phase difference of the two optical beams zero. Then, a constructive interference is established between them, resulting in a high optical signal intensity at the output port. As a result of this process, the logic operation "1 OR 1 = 1" can be accomplished.

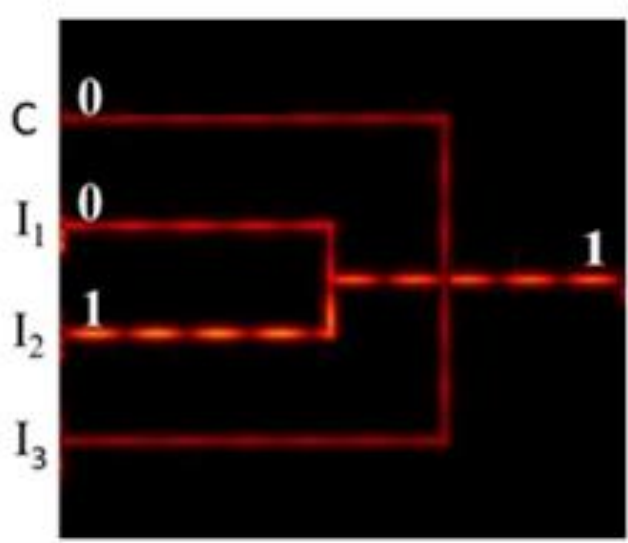

(a) logic function "0 OR 1=1"

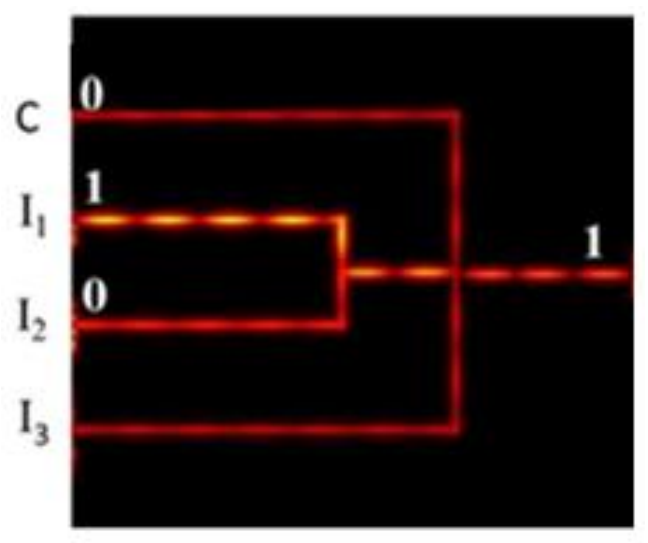

(b) logic function " 1 OR $0=1$ "

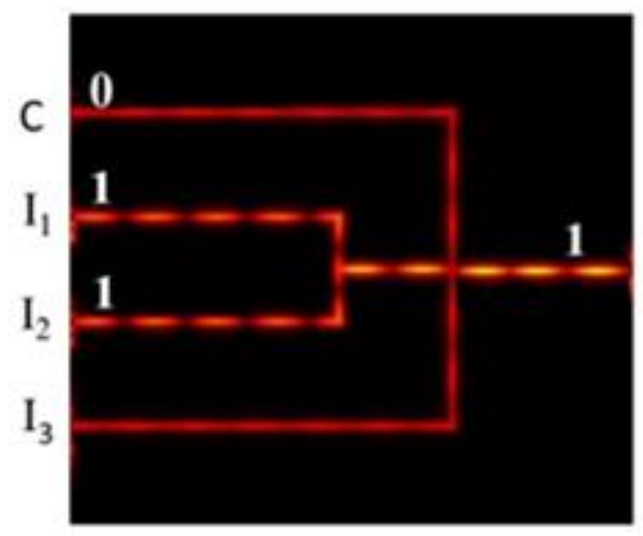

(c) logic function "1 OR 1=1"

Fig. 2 The Magnetic field distributions $\left(\left|\mathrm{H}_{\mathrm{x}}\right|\right)$ for different states of OR logic gate: (a) "0 OR $1=1$ " (b) " 1 OR $0=1$ " and (c) " 1 OR $1=1 "$.

The output field intensity diagram of the logic level "1" for the first and second proposed structures of the OR logic gate is shown in Fig. 3. By Comparing the results, it is clear that despite the Kerr nonlinear material, the field intensity has increased by $77 \mathrm{~V} / \mathrm{m}$, i.e. about $22 \%$, when the second structure is compared to the first. 


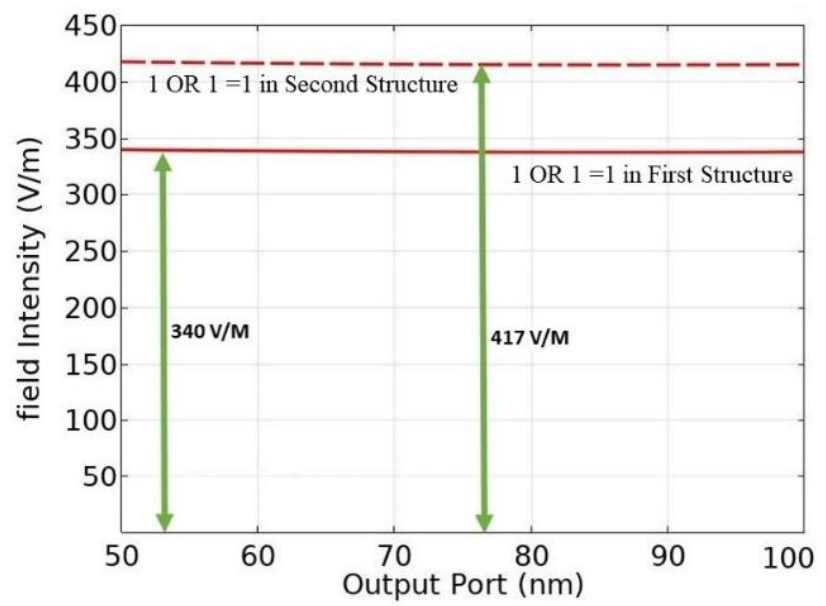

Fig. 3 The field intensity diagram at the output port of OR logic gate for the logic state of "1 OR $1=1$ " in the first proposed structure (continuous line) and the second proposed structure (dashed line)

\subsection{The AND gate performance}

To investigate the performance of AND logic gate, a structure similar to that used for the OR logic gate can be employed. The AND logic operation can be accomplished by correct definition of the threshold intensity. Table 3 shows the operation of the AND gate and its associated settings. According to the table, the logic level "1" appears in the output port only when the input port of both channels has an optical signal.

Table 3 The procedure of using gate channels to implement the AND logic function.

\begin{tabular}{|c|c|c|c|c|c|}
\hline Gate & $\begin{array}{c}\text { Input } \\
\text { Channel I }\end{array}$ & $\begin{array}{c}\text { Input } \\
\text { Channel I }\end{array}$ & $\begin{array}{c}\text { Input } \\
\text { Channel I }\end{array}$ & $\begin{array}{c}\text { Input } \\
\text { Channel C }\end{array}$ & $\begin{array}{c}\text { Output } \\
\text { Channel }\end{array}$ \\
\hline \multirow{3}{*}{ AND } & "0" & "0" & & Disable & "0" \\
\cline { 2 - 6 } & "0" & "1" & & Disable & "0" \\
\cline { 2 - 6 } & "1" & "0" & & Disable & "0" \\
\cline { 2 - 6 } & "1" & "1" & & Disable & "1" \\
\hline
\end{tabular}

The distribution of magnetic field for various logic states is shown in Fig. 4. As shown in Fig. $4(a, b)$, if an optical signal is applied to channel $I_{1}$ or channel $I_{2}$, the optical signal can be transmitted through one of the two channels and reaches the output port.

Fig. $4 \mathrm{c}$ shows that if the optical signal is applied simultaneously to the input port of channels $\mathrm{I}_{1}$ and $\mathrm{I}_{2}$, the optical signal can reach the output channel in combination. By setting a proper intensity threshold between the above two cases, the logic states of " 0 " and " 1 " can be accomplished, respectively. According to appropriate definitions, the first two processes are related to the logic function of " 1 AND $0=0$ " and "0 AND $1=0$ ", while the third process confirms that the logic function of " 1 AND $1=1$ ". The diagram of the output field intensity difference between the logic 
levels of "0" and "1" for the first and second proposed structures in the AND logic gate is shown in Fig. 5.

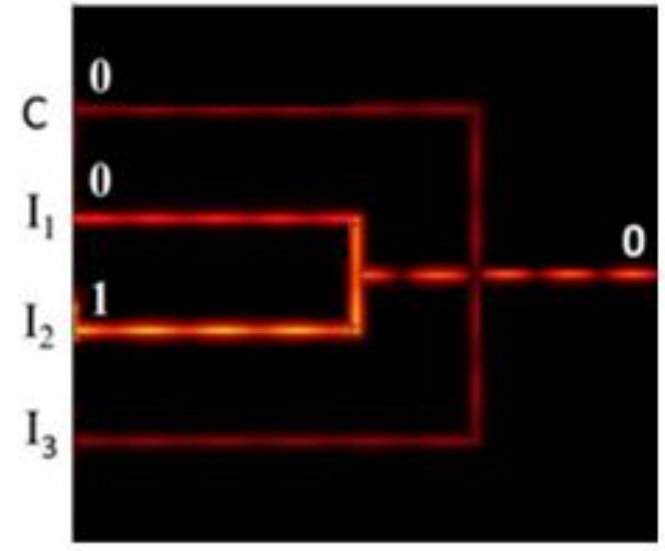

(a) logic function "0 AND 1=0"

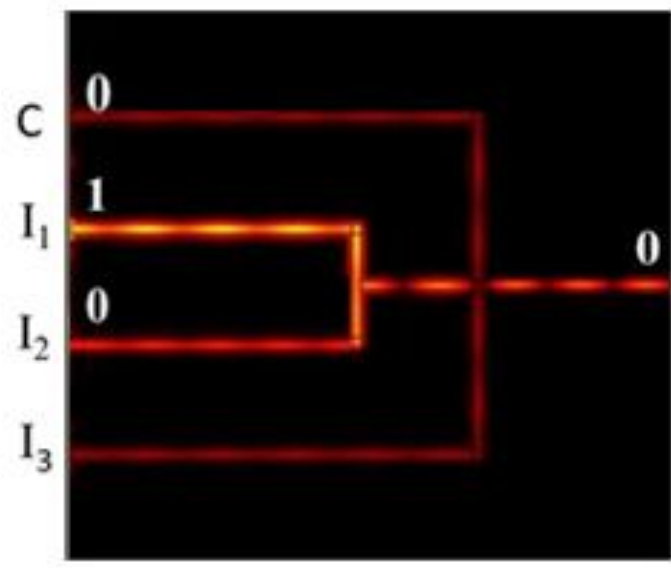

(b) logic function "1 AND 0=0"

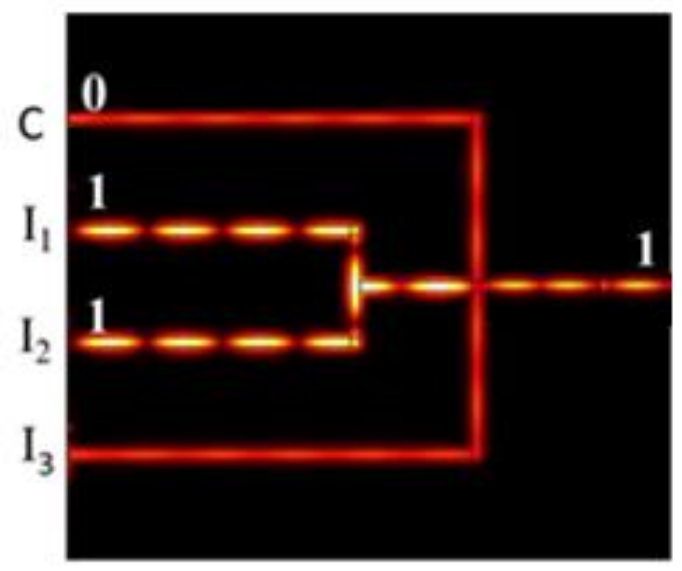

(c) logic function " 1 AND 1=1"

Fig. 4 The Magnetic field distributions $\left(\left|\mathrm{H}_{\mathrm{x}}\right|\right)$ for different states of AND logic gate: (a) "0 AND $1=0 ",(\mathbf{b})$ " 1 AND $0=0 "$ and (c) " 1 AND $1=1$ "

It can be seen that the difference of output field intensity for the state of "0 AND $1=0$ " and the state of " 1 AND $1=1$ " in the first structure is $184 \mathrm{~V} / \mathrm{m}$ (the distance between continuous blue and red lines). While the difference for the second structure has reached $317 \mathrm{~V} / \mathrm{m}$ (the distance between the blue and red dashes).

By comparing the results, it is clear that difference between the logic levels in the second structure, despite the Kerr nonlinear material, has increased about $133 \mathrm{~V} / \mathrm{m}$ relative to the first structure, i.e. $72 \%$. It can also be seen that in the second structure, the state of " 1 AND $1=1$ " increases and the state of "0 AND $1=0$ " decreases.

\subsection{The XOR gate performance}

For implementation the XOR logic operation, we need the destructive interference between optical signals of $I_{2}$ and $I_{3}$ channels. The operating principles of the XOR gate have been shown in Table 4. The magnetic field distribution for the various logic states have been shown in Fig. 6. As shown 
in Fig. 6(a,b), if a signal is entered into the input channel I2 or I3, the light beam can be transmitted via the connected channel and placed at the output port corresponding to the logic operations of " 1 XOR $0=1$ " or "0 XOR $1=1 "$ ". On the other hand, if a signal is applied simultaneously to the both input ports of channel $I_{2}$ and $I_{3}$ (Fig. 6c), the signal is transmitted and reaches the output channel in a combination form. According to what is mentioned in (Bian and Gong 2014), the difference in optical path between channels $\mathrm{I}_{2}$ and $\mathrm{I}_{3}$ is $\lambda_{\text {eff }} / 2$, which is related to the phase difference $\pi$. In this case, the destructive interference between the optical signals in channels I2 and $\mathrm{I} 3$ brings about an output with almost zero intensity at the output port, which is related to the logical operation of " 1 XOR $1=0$ ".

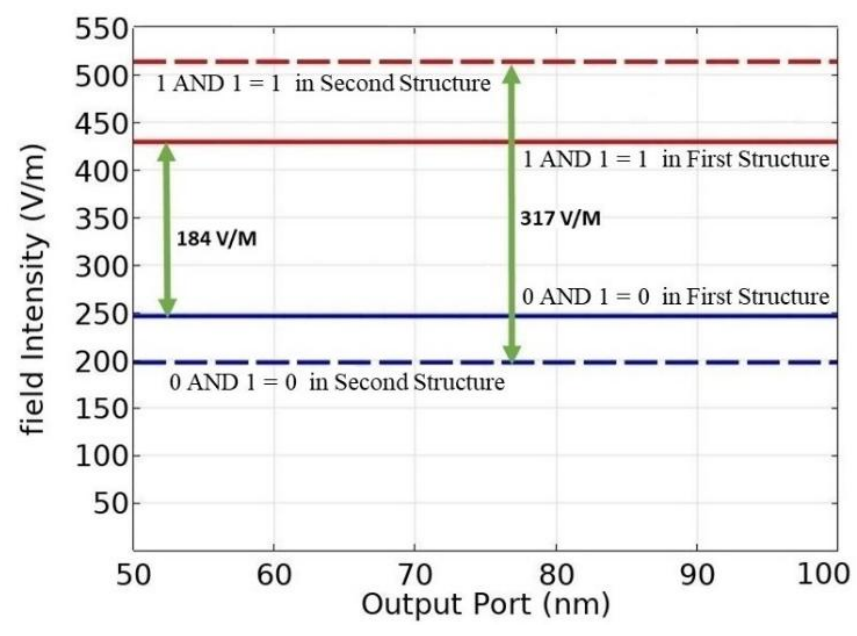

Fig. 5 The field intensity diagram at the output port of AND logic gate for two logic states " 0 AND $1=0$ " and " 1 AND 1=1" in the first proposed structure (continuous lines) and the second proposed structure (dashed lines)

Table 4 The procedure of using gate channels to implement the XOR logic function

\begin{tabular}{|c|c|c|c|c|c|}
\hline Gate & $\begin{array}{c}\text { Input } \\
\text { Channel I }\end{array}$ & $\begin{array}{c}\text { Input } \\
\text { Channel I }\end{array}$ & $\begin{array}{c}\text { Input } \\
\text { Channel I }\end{array}$ & $\begin{array}{c}\text { Input } \\
\text { Channel C }\end{array}$ & $\begin{array}{c}\text { Output } \\
\text { Channel }\end{array}$ \\
\hline \multirow{3}{*}{ XOR } & & "0" & "0" & Disable & "0" \\
\cline { 2 - 6 } & & "0" & "1" & Disable & "1" \\
\cline { 2 - 6 } & & "1" & "0" & Disable & "1" \\
\cline { 2 - 6 } & & "1" & "1" & Disable & $" 0 "$ \\
\hline
\end{tabular}

Fig. 7 shows the result of comparing the performance of the XOR gate of the first and second structures. The difference between the logic levels of " 0 " and "1" in the output of this gate has reached $131 \mathrm{~V} / \mathrm{m}$ for the first structure and $220 \mathrm{~V} / \mathrm{m}$ for the second structure. That is, the second structure compared to the first structure was able to increase $89 \mathrm{~V} / \mathrm{m}$, i.e. $67 \%$. It can be seen that the bright states have been shown a noticeable difference. Since the final state in XOR (in this diagram) results from the reaching of the $I_{3}$ channel signal to the output, it is then clear that the 
nonlinear effects occurred more strongly in the asymmetric waveguide of the $\mathrm{I}_{3}$ channel surrounded by gold and aluminum. It can be concluded from these results that by using Kerr nonlinear effect, the second structure has led to an increase in the difference of logical levels relative to the first structure.

\subsection{The NOR gate performance}

To design the NOR gate, we need a double signal intensity in the $\mathrm{C}$ control channel. The logic operation of the NOR gate can be accomplished by correctly defining the threshold intensity. Table 5 indicates the operating principles of the NOR gate. The distribution of magnetic field for different logic levels is shown in Fig. 8.

Fig. 8a illustrates the logic operation of " 0 NOR $0=1$ ", while Fig. 8(b,c,d) are related to the logic operations of "1 NOR $0=0$ ", "0 NOR $1=0$ ", and "1 NOR $1=0$ ", respectively.

According to Fig. 8a, when no signal is applied to the inputs, the control signal is placed at the output through $\mathrm{C}$ channel. So, when no signal is applied to the input, the output will be at level "1". On the other hand, if a signal is applied to the input ports of channels $I_{1}$ or $I_{2}$ (Fig. 8(b, c)), the light is transmitted through these channels and after combining with the control signal it reaches the output channel.

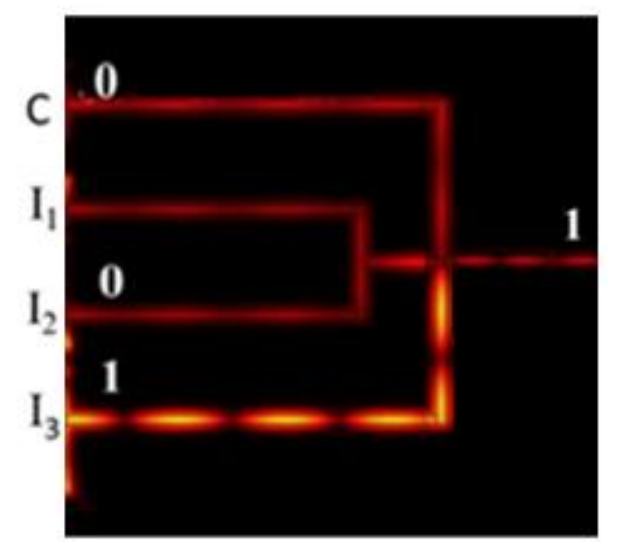

(a) logic function "0 XOR 1=1"

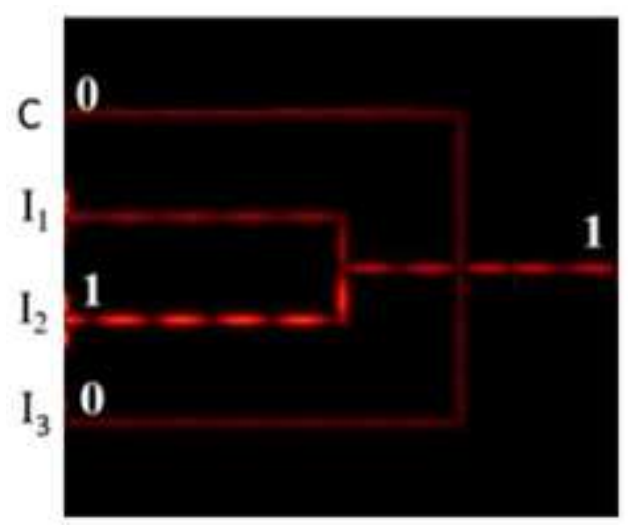

(b) logic function "1 XOR 0=1"

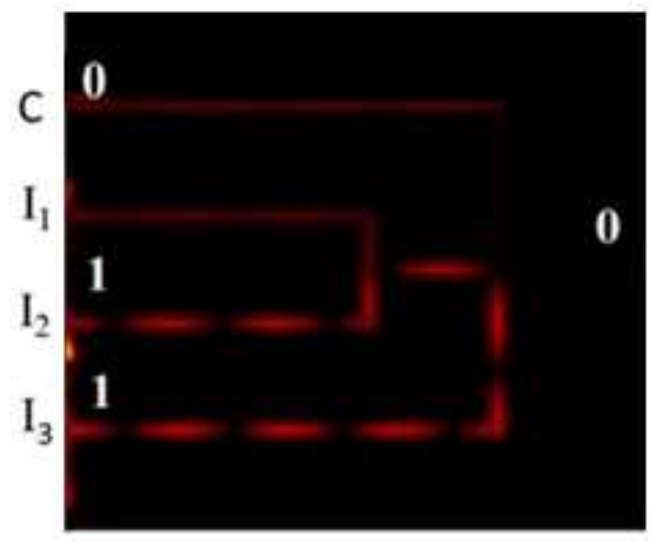

(c) logic function " 1 XOR 1=0"

Fig. 6 The Magnetic field distributions $\left(\left|\mathrm{H}_{\mathrm{x}}\right|\right)$ for different states of XOR logic gate: (a) " 0 XOR $1=1 ",(\mathbf{b})$ " 1 XOR $0=1$ " and (c) " 1 XOR $1=0$ " 


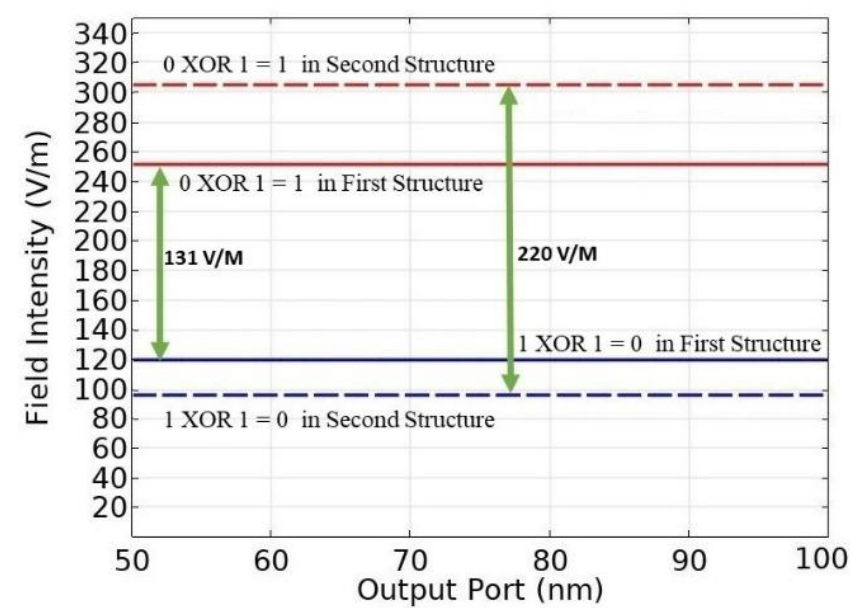

Fig. 7 The field intensity diagram at the output port of AND logic gate for two logic states " 0 XOR $1=1$ " and " 1 XOR $1=0$ " in the first proposed structure (continuous line) and the second proposed structure (dashed line)

In this case, there is a destructive interference between the signals of channels $I_{1}$ or $I_{2}$ with the control channel, and because the intensity of the control signal is twice of the input signals, the signal intensity at the output is less than that of control signal intensity, and of course more than zero.

Table 5 The procedure of using gate channels to implement the NOR logic function

\begin{tabular}{|c|c|c|c|c|c|}
\hline Gate & $\begin{array}{c}\text { Input } \\
\text { Channel } \mathrm{I}_{1}\end{array}$ & $\begin{array}{c}\text { Input } \\
\text { Channel } \mathrm{I}_{2}\end{array}$ & $\begin{array}{c}\text { Input } \\
\text { Channel } \mathrm{I}_{3}\end{array}$ & $\begin{array}{c}\text { Input } \\
\text { Channel C }\end{array}$ & $\begin{array}{l}\text { Output } \\
\text { Channel }\end{array}$ \\
\hline \multirow{4}{*}{ NOR } & " 0 ") & "0" & & "1" & "1" \\
\hline & "0" & "1" & & "1" & " 0 " \\
\hline & "1" & "0" & & "1" & “0” \\
\hline & "1" & "1" & & $" 1 "$ & "0" \\
\hline
\end{tabular}

Fig. 8d shows the state in which the signal is applied simultaneously to the input ports of channels $\mathrm{I}_{1}$ and $\mathrm{I}_{2}$. Due to the complete destructive interference between the control channel and channels $\mathrm{I}_{1}$ and $\mathrm{I}_{2}$, a light with almost zero intensity appears at the output of the gate.

The employment of the second proposed structure in the NOR gate, also significantly increases the difference between the logic levels of " 0 " and "1". This is observable from the field intensity diagram of the output port in Fig. 9. The second proposed structure has been able to increase the difference between the logic levels by $138 \mathrm{~V} / \mathrm{m}$, i.e. $44 \%$, compared to the first structure. The reason of this increase in the field intensity of logic operations of "0 NOR $0=1$ " (red dashed line) is related to the control channel. In this case, the output signal is supplied through the control channel; the gold below this waveguide and the aluminum on top of it increase the nonlinear effects by combining different plasmonic modes in such asymmetric waveguide. 


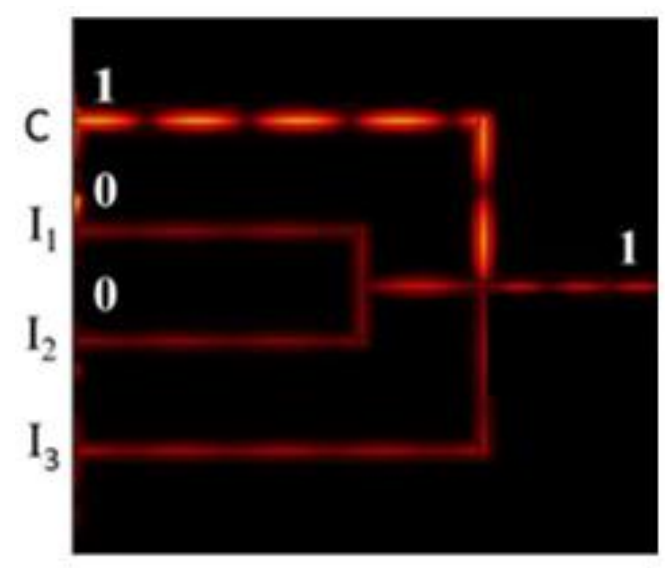

(a) logic function "0 NOR 0=1"

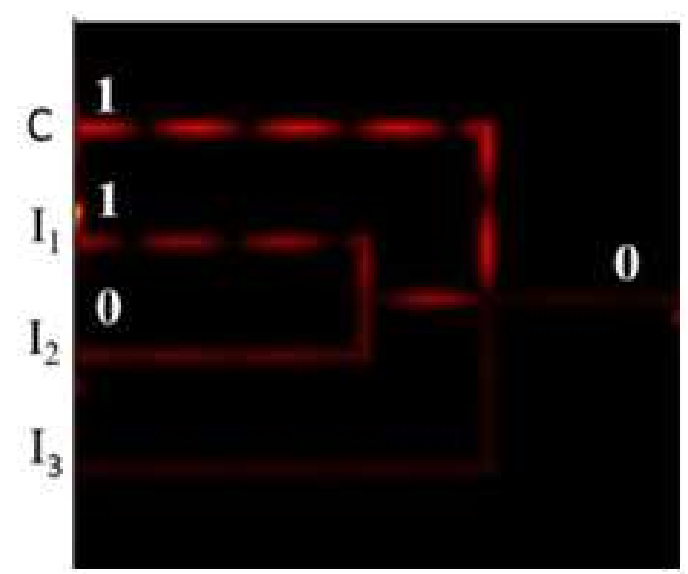

(c) logic function " 1 NOR $0=0$ "

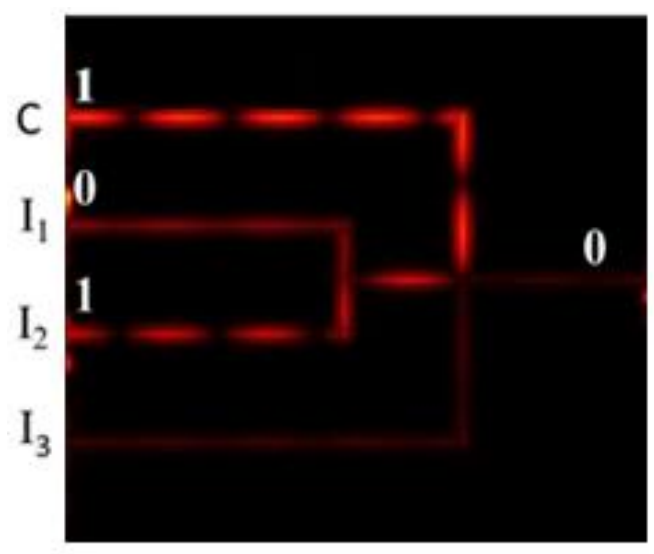

(b) logic function "0 NOR 1=0"

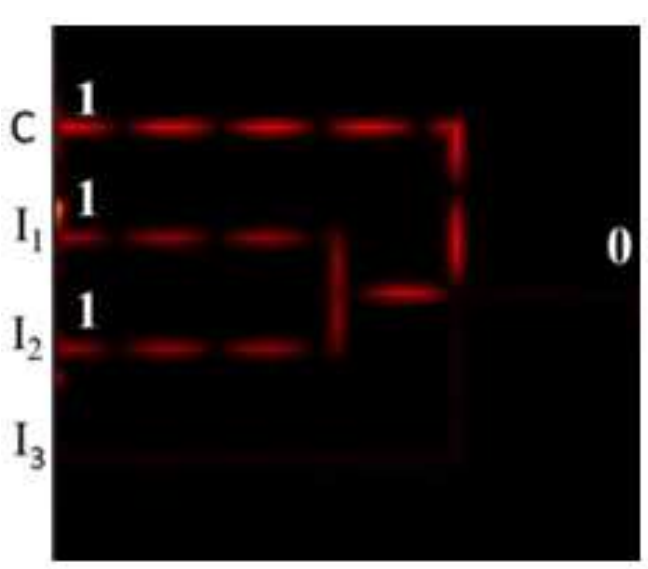

(d) logic function " 1 NOR 1=0"

Fig. 8 The Magnetic field distributions $\left(\left|\mathrm{H}_{\mathrm{x}}\right|\right)$ for different states of NOR logic gate: (a) "0 NOR $0=1 ",(\mathbf{b})$ " 0 NOR $1=0 ",(\mathbf{c})$ " 1 NOR $0=0 "$ and (d) " 1 NOR $1=0 "$.

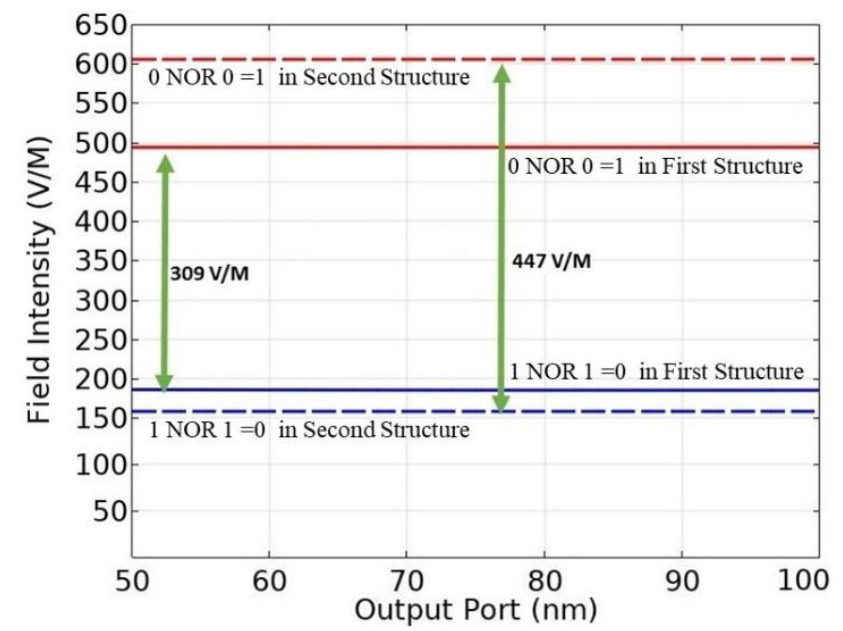

Fig. 9 The field intensity diagram at the output port of AND logic gate for two logic states " 0 NOR $0=1$ " and " 1 NOR $1=0$ " in the first proposed structure (continuous line) and the second proposed structure (dashed line) 


\section{Conclusion}

By investigating the simulation results of the proposed structure through the method of finite elements, it has been shown that by using heterogeneous metals and Kerr nonlinear material the all-optical logic gate improves its performance and it increases the level difference between the logic levels. In this paper, OR, AND, XOR and NOR logic gates were implemented. It has been shown that the linear interference between the surface plasmonic polariton modes makes the desired output at the logic gate. The advantage of the proposed structure is that a structure with fixed physical dimensions can accomplish several basic logic functions. It has been shown that the nonlinear material of Kerr increases the difference in logic level of " 0 " and " 1 " for OR gate by $23 \%$, for AND gate by $72 \%$, for XOR gate by $67 \%$, and for NOR gate by $44 \%$. The simple and compact arrangement of all-optical logic gates based on heterogeneous metal-insulator-metal plasmonic waveguides can be used in many nanophotonic devices.

\section{References}

Bian, Y., Gong, Q.: Compact all-optical interferometric logic gates based on one-dimensional metal insulator-metal structures. Optics Communications 313, 27-35 (2014)

Bian, Y., Gong, Q.: Optical performance of one-dimensional hybrid metal-insulator-metal structures at telecom wavelength. Optics Communications 308, 30-35 (2013)

Caulfield, H.J., Dolev, S.: The role of optics in computing. Nature Photonics 4, 405 (2010)

Dionne, J.A., Sweatlock, L.A., Atwater, H.A., Polman, A.: Plasmon slot waveguides: Towards chip-scale propagation with subwavelength-scale localization. Physical Review B 73, 035407 (2006)

Fu, Y., Hu. X., Gong, Q.: Silicon photonic crystal all-optical logic gates. Physics Letters A 377, 329-333 (2013)

Ghorbani, S., Sadeghi, M., Adelpour, Z.: Investigation and analysis of a tunable plasmonic filter based on the Kerr nonlinear effect of a gold nanocomposite. Laser Physics 30(8), 086201 (2020)

$\mathrm{Li}, \mathrm{Z}$., Chen, Z., Li, B.: Optical pulse controlled all-optical logic gates in SiGe/Si multimode interference. Optics express 13(3), 1033-1038 (2005)

Lu, C., Hu, X., Yue, S., Fu, Y., Yang, H., Gong, Q.: Ferroelectric hybrid plasmonic waveguide for alloptical logic gate applications. Plasmonics 8, 749-754 (2013)

Lu, H., Liu, X., Wang, L., Gong, Y., Mao, D.: Ultrafast all-optical switching in nanoplasmonic waveguide with Kerr nonlinear resonator. Optics Express 19(4), 2910-2915 (2011)

Moradipour, M., Kazemi-Esfeh, P., Alaie, Z.: Investigating the plasmonic effect on half adder and full adder based on photonic crystal. Optical and Quantum Electronics 53(66), 1-17 (2021)

Onuki, T., Watanabe, Y., Nishio, K., Tsuchiya, T., Tani, T., Tokizaki, T.: Propagation of surface plasmon polariton in nanometre-sized metal-clad optical waveguides. Journal of microscopy 210(3), 284-287 (2003)

Pan, D., Wei, H., Xu, H.: Optical interferometric logic gates based on metal slot waveguide network realizing whole fundamental logic operations. Optics Express 21(8), 9556-9562 (2013)

Papaioannou, M., Plum, E., Valente, J., Rogers, E.T.F., Zheludev, N. I.: Two-dimensional control of light with light on metasurfaces. Light: Science \& Applications 5, e16070 (2016)

Sankar, P., Philip, R.: Nonlinear optical properties of nanomaterials, Characterization of nanomaterials. Woodhead Publishing, pp. 301-334.17 (2018)

Xu, Y., Wang, X., Deng, H., Guo, K.: Tunable all-optical plasmonic rectifier in nanoscale metal-insulatormetal waveguides. Optics Letters 39(20), 5846-5849 (2014)

Zhang, Y., Zhang, Y., Li, B.: Optical switches and logic gates based on self-collimated beams in two dimensional photonic crystals. Optics Express 15(15) 9287-9292 (2007) 